\title{
Original
}

\section{ADIPOQ polymorphisms are associated with insulin resistance in Japanese women}

\author{
Aya Kitamoto ${ }^{1)}$, Takuya Kitamoto ${ }^{1)}$, Rina $\mathrm{So}^{2)}$, Tomoaki Matsuo ${ }^{2)}$, Yoshio Nakata ${ }^{2)}$, Hideyuki Hyogo ${ }^{3)}$, \\ Hidenori Ochi ${ }^{3}$, , Takahiro Nakamura ${ }^{4)}$, Seika Kamohara ${ }^{5)}$, Nobuyuki Miyatake ${ }^{6}$, Kazuaki Kotani $^{7)}$, Ikuo Mineo ${ }^{8)}$, \\ Jun Wada ${ }^{9)}$, Yuji Ogawa ${ }^{10)}$, Masato Yoneda ${ }^{10)}$, Atsushi Nakajima ${ }^{10)}$, Tohru Funahashi ${ }^{11)}$, Shigeru Miyazaki ${ }^{12)}$, \\ Katsuto Tokunaga ${ }^{13)}$, Hiroaki Masuzaki ${ }^{14)}$, Takato Ueno ${ }^{15)}$, Kazuaki Chayama ${ }^{3)}$, Kazuyuki Hamaguchi ${ }^{16)}$, \\ Kentaro Yamada ${ }^{17)}$, Toshiaki Hanafusa ${ }^{18)}$, Shinichi Oikawa ${ }^{19)}$, Toshiie Sakata ${ }^{20)}$, Kiyoji Tanaka ${ }^{2)}$, \\ Yuji Matsuzawa $^{7)}$ and Kikuko Hotta ${ }^{1)}$ \\ ${ }^{1)}$ Pharmacogenomics, Kyoto University Graduate School of Medicine, Kyoto 606-8501, Japan \\ ${ }^{2)}$ Graduate School of Comprehensive Human Sciences, University of Tsukuba, Tsukuba 305-8574, Japan \\ ${ }^{3)}$ Department of Gastroenterology and Metabolism, Institute of Biomedical and Health Sciences, Hiroshima University, Hiroshima 734-8551, Japan \\ 4) Laboratory for Mathematics, National Defense Medical College, Tokorozawa 359-8513, Japan \\ ${ }^{5)}$ Health Science University, Yamanashi 401-0380, Japan \\ 6) Department of Hygiene, Faculty of Medicine, Kagawa University, Kagawa 761-0793, Japan \\ ${ }^{7)}$ Department of Metabolic Medicine, Graduate School of Medicine, Osaka University, Suita 565-0871, Japan \\ 8) Otemae Hospital, Osaka 540-0008, Japan \\ 9) Department of Medicine and Clinical Science, Okayama University Graduate School of Medicine, Dentistry and Pharmaceutical Sciences, \\ Okayama 700-8558, Japan \\ ${ }^{10)}$ Department of Gastroenterology and Hepatology, Yokohama City University School of Medicine, Yokohama 236-0004, Japan \\ ${ }^{11)}$ Department of Metabolism and Atherosclerosis, Graduate School of Medicine, Osaka University, Suita 565-0871, Japan \\ ${ }^{12)}$ Tokyo Postal Services Agency Hospital, Tokyo 102-8798, Japan \\ ${ }^{13)}$ Itami City Hospital, Itami 664-8540, Japan \\ ${ }^{14)}$ Division of Endocrinology, Diabetes and Metabolism, Hematology, Rheumatology Graduate School of Medicine, University of the Ryukyus, \\ Okinawa 903-0215, Japan \\ ${ }^{15)}$ Research Center for Innovative Cancer Therapy, Kurume University, Kurume 830-0011, Japan \\ ${ }^{16)}$ Department of Practical Nursing Science, Faculty of Medicine, Oita University, Oita 879-5593, Japan \\ ${ }^{17)}$ Division of Endocrinology and Metabolism, Department of Medicine, Kurume University, Kurume 830-0011, Japan \\ ${ }^{18)}$ Department of Internal Medicine (I), Osaka Medical College, Takatsuki 569-8686, Japan \\ ${ }^{19)}$ Director of Diabetes and Lifestyle Disease Center, Fukujuji Hospital, Tokyo 204-8522, Japan \\ ${ }^{20)}$ Department of Internal Medicine 1, Faculty of Medicine, Oita University, Oita 879-5593, Japan
}

\begin{abstract}
Visceral fat accumulation contributes to the development of insulin resistance, leading to metabolic syndrome. Adiponectin provides a link between visceral fat accumulation and insulin resistance. In addition to environmental factors, genetic factors play important roles in visceral fat accumulation and circulating adiponectin levels. Genome-wide association studies (GWASs) have identified genetic variations in the adiponectin, $\mathrm{C} 1 \mathrm{Q}$ and collagen domain containing $(A D I P O Q)$ gene that are associated with adiponectin levels. In this study, we investigated whether $A D I P O Q$ single nucleotide polymorphisms (SNPs) were associated with visceral fat accumulation and insulin resistance. We measured the visceral fat area (VFA) by computed tomography (CT) and examined the presence of the insulin resistance-related phenotype (fasting plasma glucose, fasting insulin, and homeostasis model assessment-insulin resistance [HOMA-IR]) in a set of Japanese individuals (731 men and 864 women) who were genotyped for seven ADIPOQ SNPs reported by recent GWASs (namely, rs6810075, rs10937273, rs1648707, rs864265, rs182052, rs17366568, and rs6773957). SNPs associated with the phenotype $(P<0.05)$ were then evaluated by association analysis using a second set of the study participants ( 383 men and 510 women). None of the SNPs was associated with body mass index (BMI) or VFA in men or women. However, the adiponectin-decreasing alleles of rs 10937273 and rs1648707 were significantly associated with HOMA-IR $(P=0.0030$ and $P=0.00074$, respectively $)$ in women, independently of BMI. These SNPs were significantly associated with decreased adiponectin levels in women. Our results suggested that rs10937273 and rs1648707 may affect insulin sensitivity by regulating adiponectin production by adipose tissue in women.
\end{abstract}

Key words: $A D I P O Q$, Single nucleotide polymorphism, Visceral adipose tissue, Insulin resistance, Adiponectin

ADIPOSE TISSUE is necessary for energy homeostasis; however, excess accumulation of adipose tisSubmitted Dec.16, 2014; Accepted Mar.10, 2015 as EJ14-0574 Released online in J-STAGE as advance publication Mar. 31, 2015 Correspondence to: Kikuko Hotta, M.D., Ph.D., Pharmacogenomics, Kyoto University Graduate School of Medicine, YoshidaKonoecho, Sakyo-ku, Kyoto 606-8501, Japan E-mail: kikukoh@kuhp.kyoto-u.ac.jp sue, especially visceral fat, contributes to the development of insulin resistance, leading to type 2 diabetes, dyslipidemia, and hypertension, a condition known as metabolic syndrome [1]. Adiponectin provides a critical link between visceral fat accumulation and insulin resistance [2-5]. Circulating adiponectin levels are decreased in parallel with increased visceral fat, 
causing insulin resistance and type 2 diabetes $[4,5]$. Recently, genome-wide association studies (GWASs) have indicated that adiponectin levels are also determined by genetic variations, in addition to visceral fat accumulation [6-13]. One of the most important genetic factors determining adiponectin level is the adiponectin, C1Q and collagen domain containing ( $A D I P O Q$ ) gene itself; polymorphisms in the $A D I P O Q$ gene have been reported to be associated with insulin resistance and type 2 diabetes, in addition to adiponectin levels $[8,11,13,14]$.

In addition to mediating insulin resistance, visceral fat accumulation plays a central role in metabolic syndrome $[1,2]$. Visceral fat is evaluated by waist circumference, waist-hip ratio, bioelectrical impedance, or more precisely, measurement of visceral fat area (VFA) using computed tomography (CT) $[1,15]$. Numerous studies have shown that VFA is influenced by genetic loci [16-21]. However, despite the observation that $A D I P O Q$ is a promising candidate gene for visceral fat accumulation [22, 23], few studies have reported a link between $A D I P O Q$ polymorphisms and VFA. Moreover, associations between $A D I P O Q$ polymorphisms and insulin resistance have not been definitively established $[8,11,13,14,23]$.

In this study, we investigated whether $A D I P O Q$ polymorphisms were associated with VFA and/or insulin resistance, an important factor responsible for increased morbidity and mortality rates.

\section{Materials \& Methods}

\section{Participants}

The sample sizes of the first and second sets of Japanese participants were 1595 and 893 individuals, respectively. All participants visited outpatient clinics from 2002 to 2011 for routine medical checkups or treatment for metabolic abnormalities, such as obesity, hypertension, dyslipidemia, and type 2 diabetes. Patients with a disease (such as cancer, renal disease, cardiovascular disease, and hepatic failure) or receiving medication (i.e., taking insulin, corticosteroids, or chemotherapy) that would be expected to affect body weight were excluded. The first set of participants agreed to undergo CT testing (in the supine position) to determine VFA and subcutaneous fat area (SFA) at the umbilical level (L4-L5), as previously reported [24]. The values of VFA and SFA were calculated using the FatScan software program (N2system, Osaka, Japan) [24]. Clinical data were recorded at the first visit to the hospital, and the clinical characteristics of the participants are summarized in Table 1. Homeostasis

Table 1 Clinical characteristics of the participants

\begin{tabular}{|c|c|c|c|c|}
\hline & \multicolumn{2}{|c|}{1 st set } & \multicolumn{2}{|c|}{ 2nd set } \\
\hline & Men & Women & Men & Women \\
\hline $\mathrm{n}$ & 731 & 864 & 383 & 510 \\
\hline Age (years) & $\begin{array}{c}48.2 \pm 12.6 \\
(39.0,48.0,59.0)\end{array}$ & $\begin{array}{c}52.8 \pm 11.4 \\
(46.0,54.0,61.0)\end{array}$ & $\begin{array}{c}60 \pm 13.8 \\
(52.0,61.0,71.0)\end{array}$ & $\begin{array}{c}61.4 \pm 11.7 \\
(54.0,62.0,70.0)\end{array}$ \\
\hline BMI $\left(\mathrm{kg} / \mathrm{m}^{2}\right)$ & $\begin{array}{c}29.7 \pm 5.8 \\
(26.3,29.0,32.0)\end{array}$ & $\begin{array}{c}28.1 \pm 5.3 \\
(24.8,27.1,30.7)\end{array}$ & $\begin{array}{c}26.2 \pm 4.7 \\
(23.0,25.5,28.4)\end{array}$ & $\begin{array}{c}25.5 \pm 4.1 \\
(22.7,25.2,27.9)\end{array}$ \\
\hline $\operatorname{VFA}\left(\mathrm{cm}^{2}\right)$ & $\begin{array}{c}152.5 \pm 66.3 \\
(107.6,142.7,191.0)\end{array}$ & $\begin{array}{c}104.7 \pm 54.4 \\
(65.4,97.5,136.2)\end{array}$ & - & - \\
\hline $\operatorname{SFA}\left(\mathrm{cm}^{2}\right)$ & $\begin{array}{c}204.9 \pm 106.8 \\
(136.5,181.6,245.5)\end{array}$ & $\begin{array}{c}242.3 \pm 98.8 \\
(178.7,226.3,293.4)\end{array}$ & - & - \\
\hline Fasting plasma glucose $(\mathrm{mg} / \mathrm{dL})$ & $\begin{array}{c}110.6 \pm 33.5 \\
(92.0,100.0,115.0)\end{array}$ & $\begin{array}{c}109.1 \pm 35.6 \\
(90.0,97.0,112.8)\end{array}$ & $\begin{array}{c}105.6 \pm 25.7 \\
(92.0,99.0,111.5)\end{array}$ & $\begin{array}{c}99.9 \pm 19.6 \\
(89.0,95.0,104.8)\end{array}$ \\
\hline Fasting insulin $(\mu \mathrm{U} / \mathrm{mL})$ & $\begin{array}{c}13.7 \pm 16.9 \\
(6.3,9.8,15.7)\end{array}$ & $\begin{array}{c}10.8 \pm 10.3 \\
(5.0,8.1,13.3)\end{array}$ & $\begin{array}{c}12.7 \pm 18.4 \\
(3.9,6.6,13.7)\end{array}$ & $\begin{array}{c}10.6 \pm 11.7 \\
(4.6,6.7,11.6)\end{array}$ \\
\hline HOMA-IR & $\begin{array}{c}4.0 \pm 7.1 \\
(1.5,2.5,4.2)\end{array}$ & $\begin{array}{c}3.1 \pm 3.8 \\
(1.2,2.1,3.5)\end{array}$ & $\begin{array}{c}3.7 \pm 7.1 \\
(0.9,1.7,3.4)\end{array}$ & $\begin{array}{c}2.8 \pm 3.5 \\
(1.1,1.6,2.9)\end{array}$ \\
\hline Adiponectin $(\mu \mathrm{g} / \mathrm{mL})$ & $\begin{array}{c}6.1 \pm 3.1 \\
(4.2,5.5,6.9)^{*}\end{array}$ & $\begin{array}{c}9.8 \pm 5.0 \\
(6.4,8.8,11.7)^{\S}\end{array}$ & - & - \\
\hline Obesity & $608(83.2 \%)$ & $624(72.2 \%)$ & $217(56.7 \%)$ & $268(52.5 \%)$ \\
\hline Impaired glucose tolerance & $243(33.2 \%)$ & $258(29.9 \%)$ & $107(27.9 \%)$ & $92(18.0 \%)$ \\
\hline Type 2 diabetes & $152(20.8 \%)$ & $174(20.1 \%)$ & $88(23.0 \%)$ & $75(14.7 \%)$ \\
\hline Dyslipidemia & $403(55.1 \%)$ & $319(36.9 \%)$ & $157(41.0 \%)$ & $143(28.0 \%)$ \\
\hline Hypertension & $404(55.3 \%)$ & $490(56.7 \%)$ & $244(63.7 \%)$ & $288(56.5 \%)$ \\
\hline Treatment of type 2 diabetes & $98(13.4 \%)$ & $106(12.3 \%)$ & $27(7.0 \%)$ & $24(4.7 \%)$ \\
\hline
\end{tabular}


model assessment-insulin resistance (HOMA-IR) was assessed as fasting insulin $(\mu \mathrm{U} / \mathrm{mL}) \times$ fasting plasma glucose $(\mathrm{mg} / \mathrm{dL}) / 405$. Written informed consent was obtained from each participant, and the protocol was approved by Kyoto University (G374).

\section{DNA extraction and SNP genotyping}

Genomic DNA was extracted from blood samples collected from each participant using a Genomix kit (Talent Srl, Trieste, Italy). We selected seven SNPs in the $A D I P O Q$ gene that were recently identified by GWAS [6-12] and constructed Invader probes (Third Wave Technologies, Madison, WI) for these SNPs. The following seven SNPs were genotyped using Invader assays, as previously described [25], and used for analysis: rs6810075, rs10937273, rs1648707, rs864265, rs182052, rs17366568, and rs6773957. The success rate of these assays was greater than $99.0 \%$.

\section{Measurement of plasma adiponectin levels}

Plasma samples were available in 945 participants (412 men and 533 women) for the first set. Plasma total adiponectin levels were measured using an enzyme-linked immunosorbent assay (ELISA; human adiponectin ELISA kit; Otsuka Pharmaceutical Co., Tokushima, Japan).

\section{Statistical analysis}

For the additive model, we characterized the genotypes as 0,1 , or 2 , depending on the number of copies of the risk alleles. Risk alleles were defined as previously identified adiponectin-decreasing alleles [6-12]. Multiple linear regression analyses were performed to test the independent effects of each allele from each SNP on anthropometric (body mass index [BMI], VFA, SFA, and the VFA to SFA [V/S] ratio), and serum parameters (fasting plasma glucose, fasting insulin, and HOMA-IR), accounting for the effects of other variables (i.e., age and logarithmically transformed BMI). The BMI, VFA, SFA, V/S ratio, fasting glucose, fasting insulin, HOMA-IR, and adiponectin values were logarithmically transformed before performing multiple linear regression analysis. Combined $P$-values were obtained using multiple linear regression analysis by taking into account the effects of other variables (i.e., age, BMI, and participant set). The Hardy-Weinberg equilibrium was assessed using the $\chi^{2}$-test [26]. All statistical analyses were performed using R software (http:// www.r-project.org/). The program Haplo.Stats (http:// mayoresearch.mayo.edu/mayo/research/schaid_lab/ software.cfm) was used to explore associations of ADIPOQ haplotypes and HOMA-IR. Differences with $P$-values of less than $0.0071(0.05 /$ seven SNPs) were considered statistically significant.

\section{Results}

The clinical characteristics and genotypes of the participants are shown in Tables 1 and 2. All SNPs were in Hardy-Weinberg equilibrium, except rs 10937273 ( $P$ $=0.040)$ in men. The risk allele frequencies did not diverge from those reported in the HapMap database. Sexual dimorphism in fat distribution was established many years ago [27]; therefore, we independently compared SNPs with fat distribution parameters (BMI, VFA, SFA, and V/S ratio) in men and women. We performed association study using participants with a wide range of BMIs since Japanese individuals are known to have low insulin levels [28]. Multiple linear regression analyses of the anthropometric parameters with respect to the seven analyzed SNPs are shown in Table 3. No significant associations were observed. Weak associa-

Table 2 Genotypic characteristics of the first set of participants

\begin{tabular}{lcccccccc}
\hline \multirow{2}{*}{ SNP ID } & \multirow{2}{*}{$\begin{array}{c}\text { Position } \\
\text { (GRCh38) }\end{array}$} & \multirow{2}{*}{$\begin{array}{c}\text { allele1/ } \\
\text { allele2 }\end{array}$} & $\begin{array}{c}\text { Adiponectin } \\
\text { decreasing }\end{array}$ & \multicolumn{2}{c}{ Genotype } & & \multicolumn{2}{c}{ HWE $P$-value } \\
\cline { 8 - 9 } allele & & Men & Women & & Men & Women \\
\hline rs6810075 & $186,830,776$ & $\mathrm{C} / \mathrm{T}$ & $\mathrm{C}$ & $164 / 377 / 186$ & $199 / 452 / 212$ & & 0.30 & 0.16 \\
rs10937273 & $186,831,906$ & $\mathrm{~A} / \mathrm{G}$ & $\mathrm{G}$ & $79 / 359 / 291$ & $108 / 403 / 353$ & & $\mathbf{0 . 0 4 0}$ & 0.67 \\
rs1648707 & $186,833,922$ & $\mathrm{~A} / \mathrm{C}$ & $\mathrm{C}$ & $115 / 376 / 238$ & $148 / 430 / 285$ & & 0.10 & 0.51 \\
rs864265 & $186,836,503$ & $\mathrm{G} / \mathrm{T}$ & $\mathrm{T}$ & $642 / 85 / 2$ & $759 / 100 / 4$ & & 0.64 & 0.72 \\
rs182052 & $186,842,993$ & $\mathrm{~A} / \mathrm{G}$ & $\mathrm{A}$ & $163 / 377 / 191$ & $200 / 449 / 215$ & & 0.37 & 0.24 \\
rs17366568 & $186,852,664$ & $\mathrm{~A} / \mathrm{G}$ & $\mathrm{A}$ & $0 / 36 / 695$ & $1 / 44 / 819$ & & 0.49 & 0.61 \\
rs6773957 & $186,855,916$ & $\mathrm{~A} / \mathrm{G}$ & $\mathrm{G}$ & $256 / 337 / 136$ & $300 / 411 / 152$ & & 0.18 & 0.58 \\
\hline
\end{tabular}

HWE, Hardy-Weinberg equilibrium. Numbers in bold indicate $P$-values less than 0.05 . 
Table 3 Association of the seven SNPs with anthropometric parameters in men and women in the first set of participants

\begin{tabular}{|c|c|c|c|c|c|c|c|c|c|c|c|}
\hline \multirow[b]{3}{*}{ Phenotype } & \multirow[b]{3}{*}{ SNP ID } & \multicolumn{5}{|c|}{ Men } & \multicolumn{5}{|c|}{ Women } \\
\hline & & \multicolumn{3}{|c|}{ Genotypic means } & \multicolumn{2}{|c|}{ Effect per risk allele } & \multicolumn{3}{|c|}{ Genotypic means } & \multicolumn{2}{|c|}{ Effect per risk allele } \\
\hline & & 11 & 12 & 22 & $\beta$ (s.e.) & $P$-value* & 11 & 12 & 22 & $\beta$ (s.e.) & $P$-value* \\
\hline \multirow{5}{*}{$\begin{array}{l}\mathrm{BMI} \\
\left(\mathrm{kg} / \mathrm{m}^{2}\right)\end{array}$} & rs6810075 & $29.7 \pm 5.4$ & $29.6 \pm 5.9$ & $29.9 \pm 6.2$ & $0.000(0.004)$ & $1.00(0.90)$ & $28.1 \pm 5.3$ & $28.1 \pm 5.4$ & $28.3 \pm 5.1$ & $-0.002(0.004)$ & $0.55(0.54)$ \\
\hline & rs1648707 & $30.1 \pm 7.0$ & $29.7 \pm 5.9$ & $29.5 \pm 5.2$ & $-0.002(0.004)$ & $0.55(0.48)$ & $28.3 \pm 5.4$ & $28.2 \pm 5.3$ & $28.0 \pm 5.2$ & $-0.003(0.004)$ & $0.44(0.41)$ \\
\hline & rs 864265 & $29.7 \pm 5.8$ & $29.8 \pm 6.3$ & $34.2 \pm 1.2$ & $0.004(0.008)$ & $0.63(0.60)$ & $28.0 \pm 5.3$ & $28.7 \pm 4.6$ & $33.0 \pm 6.8$ & $0.016(0.007)$ & $0.029(0.037)$ \\
\hline & rs 182052 & $29.7 \pm 5.4$ & $29.6 \pm 5.9$ & $29.8 \pm 6.1$ & $0.000(0.004)$ & $0.98(0.84)$ & $28.1 \pm 5.3$ & $28.1 \pm 5.4$ & $28.3 \pm 5.1$ & $-0.001(0.004)$ & $0.69(0.71)$ \\
\hline & rs6773957 & $30.0 \pm 6.1$ & $29.6 \pm 6.0$ & $29.3 \pm 4.8$ & $-0.002(0.004)$ & $0.59(0.43)$ & $28.2 \pm 4.9$ & $28.0 \pm 5.4$ & $28.2 \pm 5.6$ & $-0.001(0.004)$ & $0.70(0.75)$ \\
\hline \multirow{5}{*}{$\begin{array}{l}\text { VFA } \\
\left(\mathrm{cm}^{2}\right)\end{array}$} & rs6810075 & $151.5 \pm 60.3$ & $151.2 \pm 66.9$ & $155.7 \pm 70.3$ & $0.000(0.010)$ & $0.98(0.98)$ & $105.3 \pm 57.3$ & $104.9 \pm 55.9$ & $104.0 \pm 48.4$ & $0.000(0.010)$ & $0.96(0.96)$ \\
\hline & rs10937273 & $153.8 \pm 64.3$ & $152.8 \pm 69.5$ & $151.2 \pm 62.8$ & $0.003(0.010)$ & $0.77(0.74)$ & $102.6 \pm 50.7$ & $103.1 \pm 54.2$ & $107.2 \pm 55.7$ & $0.008(0.010)$ & $0.42(0.44)$ \\
\hline & rs 1648707 & $155.9 \pm 66.0$ & $152.6 \pm 69.1$ & $150.6 \pm 61.8$ & $0.000(0.010)$ & $0.97(0.93)$ & $102.9 \pm 49.1$ & $104.7 \pm 55.3$ & $105.9 \pm 55.9$ & $0.009(0.010)$ & $0.34(0.35)$ \\
\hline & rs864265 & $152.1 \pm 66.1$ & $154.5 \pm 68.7$ & $176.3 \pm 38.9$ & $0.007(0.020)$ & $0.72(0.74)$ & $104.0 \pm 54.8$ & $109.2 \pm 52.2$ & $112.2 \pm 38.9$ & $-0.007(0.020)$ & $0.73(0.71)$ \\
\hline & rs 182052 & $149.9 \pm 59.5$ & $152.2 \pm 67.1$ & $155.1 \pm 70.1$ & $-0.001(0.010)$ & $0.91(0.97)$ & $104.7 \pm 57.5$ & $105.3 \pm 55.7$ & $103.5 \pm 48.7$ & $-0.001(0.010)$ & $0.89(0.89)$ \\
\hline \multirow{6}{*}{$\begin{array}{l}\text { SFA } \\
\left(\mathrm{cm}^{2}\right)\end{array}$} & rs 10937273 & $220.0 \pm 129.7$ & $201.6 \pm 105.12$ & $205.2 \pm 102.3$ & $0.007(0.008)$ & $0.38(0.35)$ & $242.5 \pm 99.7$ & $236.7 \pm 96.3$ & $248.7 \pm 101.2$ & $0.008(0.006)$ & $0.16(0.13)$ \\
\hline & rs1648707 & $221.8 \pm 129.7$ & $201.3 \pm 103.4$ & $202.7 \pm 99.8$ & $0.002(0.007)$ & $0.80(0.74)$ & $238.9 \pm 96.3$ & $239.6 \pm 95.8$ & $248.7 \pm 104.3$ & $0.013(0.006)$ & $0.027(0.022)$ \\
\hline & rs864265 & $202.8 \pm 104.8$ & $219.9 \pm 122.4$ & $229.3 \pm 58.3$ & $0.021(0.015)$ & $0.15(0.16)$ & $241.6 \pm 98.9$ & $241.7 \pm 87.7$ & $271.5 \pm 78.8$ & $-0.015(0.012)$ & $0.18(0.20)$ \\
\hline & rs182052 & $204.4 \pm 100.5$ & $200.5 \pm 100.7$ & $214.3 \pm 122.8$ & $0.000(0.007)$ & $0.97(0.89)$ & $250.1 \pm 104.2$ & $239.6 \pm 99.8$ & $240.8 \pm 91.3$ & $0.008(0.006)$ & $0.14(0.14)$ \\
\hline & rs 17366568 & - & $227.2 \pm 135.5$ & $203.8 \pm 105.1$ & $0.017(0.023)$ & $0.46(0.40)$ & 197.4 & $226.7 \pm 83.0$ & $243.2 \pm 99.6$ & $-0.006(0.017)$ & $0.75(0.70)$ \\
\hline & rs6773957 & $211.9 \pm 113.4$ & $201.4 \pm 104.9$ & $201.1 \pm 98.9$ & $0.001(0.007)$ & $0.93(0.79)$ & $241.9 \pm 92.9$ & $238.1 \pm 96.7$ & $255.6 \pm 113.7$ & $0.010(0.006)$ & $0.086(0.091)$ \\
\hline \multirow{5}{*}{ V/S ratio } & rs6810075 & $0.83 \pm 0.41$ & $0.85 \pm 0.40$ & $0.85 \pm 0.43$ & $-0.001(0.01)$ & $0.92(0.94)$ & $0.46 \pm 0.26$ & $0.47 \pm 0.28$ & $0.46 \pm 0.23$ & $-0.008(0.011)$ & $0.46(0.45)$ \\
\hline & rs 10937273 & $0.82 \pm 0.38$ & $0.86 \pm 0.43$ & $0.83 \pm 0.39$ & $-0.002(0.011)$ & $0.82(0.84)$ & $0.45 \pm 0.21$ & $0.46 \pm 0.26$ & $0.47 \pm 0.27$ & $0.000(0.011)$ & $0.99(0.93)$ \\
\hline & rs1648707 & $0.85 \pm 0.44$ & $0.86 \pm 0.41$ & $0.84 \pm 0.40$ & $0.000(0.011)$ & $0.98(0.97)$ & $0.46 \pm 0.20$ & $0.47 \pm 0.28$ & $0.46 \pm 0.26$ & $-0.004(0.011)$ & $0.68(0.66)$ \\
\hline & rs 864265 & $0.85 \pm 0.41$ & $0.84 \pm 0.45$ & $0.82 \pm 0.38$ & $-0.017(0.022)$ & $0.44(0.43)$ & $0.46 \pm 0.26$ & $0.48 \pm 0.28$ & $0.42 \pm 0.12$ & $0.015(0.022)$ & $0.50(0.58)$ \\
\hline & rs 182052 & $0.83 \pm 0.40$ & $0.86 \pm 0.40$ & $0.85 \pm 0.44$ & $-0.001(0.010)$ & $0.92(0.95)$ & $0.46 \pm 0.26$ & $0.47 \pm 0.28$ & $0.46 \pm 0.23$ & $-0.010(0.011)$ & $0.34(0.34)$ \\
\hline
\end{tabular}

Data are expressed as means \pm SDs. Allele1, allele2, and the tested (adiponectin-decreasing) allele of each SNP are indicated in Table 2. The effect sizes and $P$-values are derived from linear regression analysis. Log-transformed BMIs and V/S ratios were adjusted for age. Log-transformed VFA and SFA were adjusted for age and log-transformed BMI. *In parentheses, $P$-values further adjusted for the treatment for type 2 diabetes. 11, allele1/allele1; 12 , allele1/allele2; 22, allele2/allele2. Numbers in bold indicate $P$-values less than 0.05 .

tions between the risk allele of rs 864265 and increased BMI, as well as that of rs1648707 with increased SFA were observed in women. The risk allele of rs6773957 tended to be associated with decreases in VFA and the $\mathrm{V} / \mathrm{S}$ ratio in women.

Next, we examined insulin resistance-related parameters in men and women (Table 4). Risk alleles of five SNPs exhibited weak associations $(P<0.05)$ with insulin resistance-related parameters in men or women: rs6810075 and rs10937273 with HOMA-IR, rs1648707 with insulin and HOMA-IR in women, and rs6773957 with fasting plasma glucose in men. SNPs associated with the insulin resistance-related phenotype $(P<0.05)$ were then subjected to association analysis using the second set of participants (383 men and 510 women), and serum parameters (fasting plasma glucose, fasting insulin, and HOMA-IR) and BMI were analyzed (Table 5). Associations of two SNPs (rs10937273 and rs1648707) with HOMA-IR and rs1648707 with fasting insulin were replicated in women, and the combined $P$-values were significant. Haplotype analysis revealed that the GC haplotype composed of rs10937273 and rs1648707 was most significantly associated with HOMA-IR in women $(P=$ 0.0011; Supplementary Table 1).

We measured the plasma adiponectin levels in 945 individuals (412 men and 533 women) of the first set and replicated the significant association of rs 10937273 in men and women, and rs1648707 in women with decreased adiponectin levels (Table 4). 
Table 4 Association of the seven SNPs with serum parameters in men and women in the first set of participants

\begin{tabular}{|c|c|c|c|c|c|c|c|c|c|c|c|}
\hline \multirow[b]{3}{*}{ Phenotype } & \multirow[b]{3}{*}{ SNP ID } & \multicolumn{5}{|c|}{ Men } & \multicolumn{5}{|c|}{ Women } \\
\hline & & \multicolumn{3}{|c|}{ Genotypic means } & \multicolumn{2}{|c|}{ Effect per risk allele } & \multicolumn{3}{|c|}{ Genotypic means } & \multicolumn{2}{|c|}{ Effect per risk allele } \\
\hline & & 11 & 12 & 22 & $\beta$ (s.e.) & $P$-value* & 11 & 12 & 22 & $\beta$ (s.e.) & $P$-value* \\
\hline \multirow{7}{*}{$\begin{array}{l}\text { Fasting } \\
\text { plasma } \\
\text { glucose } \\
(\mathrm{mg} / \mathrm{dL})\end{array}$} & rs6810075 & $108.6 \pm 28.1$ & $111.4 \pm 36.3$ & $111.3 \pm 32.3$ & $-0.005(0.005)$ & $0.39(0.12)$ & $109.8 \pm 37.7$ & $108.7 \pm 33.4$ & $109.5 \pm 38.3$ & $0.003(0.005)$ & $0.59(0.54)$ \\
\hline & rs10937273 & $105.2 \pm 18.9$ & $112.4 \pm 36.6$ & $110.0 \pm 32.6$ & $0.001(0.006)$ & $0.80(0.95)$ & $111.7 \pm 43.7$ & $107.4 \pm 32.4$ & $110.3 \pm 36.4$ & $0.004(0.005)$ & $0.49(0.61)$ \\
\hline & rs1648707 & $108.6 \pm 24.8$ & $112.0 \pm 37.3$ & $109.4 \pm 30.9$ & $-0.001(0.005)$ & $0.79(0.45)$ & $110.0 \pm 39.6$ & $108.1 \pm 33.1$ & $110.4 \pm 37.2$ & $0.005(0.005)$ & $0.32(0.32)$ \\
\hline & rs 864265 & $110.2 \pm 33.1$ & $113.5 \pm 37.2$ & $126.0 \pm 48.1$ & $0.011(0.011)$ & $0.33(0.18)$ & $109.5 \pm 36.3$ & $105.6 \pm 28.9$ & $140.8 \pm 37.7$ & $-0.009(0.01)$ & $0.39(0.22)$ \\
\hline & rs 182052 & $110.6 \pm 37.1$ & $110.5 \pm 32.8$ & $111.0 \pm 31.9$ & $-0.002(0.005)$ & $0.68(0.23)$ & $109.6 \pm 37.7$ & $108.8 \pm 33.4$ & $109.5 \pm 38.1$ & $0.002(0.005)$ & $0.70(0.59)$ \\
\hline & rs17366568 & - & $112.1 \pm 36.0$ & $110.6 \pm 33.4$ & $0.006(0.017)$ & $0.73(0.77)$ & 169 & $108.6 \pm 41.6$ & $109.1 \pm 35.2$ & $0.004(0.016)$ & $0.81(0.60)$ \\
\hline & rs6773957 & $107.3 \pm 27.7$ & $111.5 \pm 35.6$ & $115.0 \pm 38.0$ & $0.011(0.005)$ & $\mathbf{0 . 0 3 2}(0.16)$ & $110.3 \pm 36.0$ & $109.2 \pm 34.7$ & $106.9 \pm 37.4$ & $-0.007(0.005)$ & $0.20(0.18)$ \\
\hline \multirow{7}{*}{$\begin{array}{l}\text { Insulin } \\
(\mu \mathrm{U} / \mathrm{mL})\end{array}$} & rs6810075 & $12.4 \pm 10.5$ & $12.5 \pm 10.4$ & $17.5 \pm 28.4$ & $-0.042(0.016)$ & $0.010(0.0090)$ & $11.7 \pm 12.5$ & $11.0 \pm 10.2$ & $9.8 \pm 7.9$ & $0.029(0.015)$ & $0.056(0.058)$ \\
\hline & rs10937273 & $14.0 \pm 14.2$ & $14.9 \pm 21.1$ & $12.3 \pm 10.9$ & $-0.021(0.017)$ & $0.22(0.22)$ & $9.7 \pm 7.4$ & $10.6 \pm 10.1$ & $11.4 \pm 11.2$ & $0.030(0.016)$ & $0.059(0.061)$ \\
\hline & rs1648707 & $17.6 \pm 31.4$ & $13.7 \pm 13.9$ & $11.9 \pm 9.8$ & $-0.036(0.017)$ & $0.031(0.029)$ & $9.7 \pm 7.4$ & $10.7 \pm 9.9$ & $11.6 \pm 11.9$ & $0.031(0.015)$ & $\mathbf{0 . 0 4 4}(0.051)$ \\
\hline & rs 864265 & $13.1 \pm 12.5$ & $18.5 \pm 34.8$ & 12 & $0.065(0.033)$ & $0.051(\mathbf{0 . 0 4 6})$ & $11.0 \pm 10.7$ & $9.6 \pm 6.7$ & $15.2 \pm 8.3$ & $-0.017(0.031)$ & $0.58(0.64)$ \\
\hline & rs182052 & $12.2 \pm 10.5$ & $12.6 \pm 10.4$ & $17.3 \pm 28$ & $-0.045(0.016)$ & $0.0062(0.0050)$ & $11.5 \pm 12.4$ & $11.0 \pm 10.2$ & $9.9 \pm 7.9$ & $0.023(0.015)$ & $0.13(0.13)$ \\
\hline & rs17366568 & - & $15.4 \pm 23.7$ & $13.6 \pm 16.6$ & $0.003(0.054)$ & $0.95(0.98)$ & 21.8 & $10.3 \pm 8.9$ & $10.9 \pm 10.4$ & $0.012(0.045)$ & $0.79(0.78)$ \\
\hline & rs6773957 & $13.4 \pm 11.7$ & $12.9 \pm 18.3$ & $16.3 \pm 21.5$ & $0.019(0.016)$ & $0.22(0.26)$ & $11.6 \pm 11.8$ & $10.2 \pm 9.3$ & $11.1 \pm 9.8$ & $-0.009(0.015)$ & $0.54(0.56)$ \\
\hline \multirow[t]{7}{*}{ HOMA-IR } & rs6810075 & $3.4 \pm 3.5$ & $3.6 \pm 3.6$ & $5.4 \pm 12.7$ & $-0.047(0.019)$ & $0.011(0.0071)$ & $3.3 \pm 4.5$ & $3.1 \pm 3.9$ & $2.6 \pm 2.5$ & $0.036(0.017)$ & $0.038(0.036)$ \\
\hline & rs10937273 & $3.9 \pm 4.5$ & $4.5 \pm 9.3$ & $3.5 \pm 3.9$ & $-0.023(0.020)$ & $0.25(0.24)$ & $2.7 \pm 2.5$ & $3.0 \pm 3.9$ & $3.2 \pm 3.9$ & $0.037(0.018)$ & $0.040(0.039)$ \\
\hline & rs 1648707 & $5.6 \pm 15.0$ & $3.9 \pm 4.6$ & $3.3 \pm 3.4$ & $-0.040(0.019)$ & $0.036(0.028)$ & $2.7 \pm 2.5$ & $3.0 \pm 3.8$ & $3.3 \pm 4.2$ & $0.040(0.017)$ & $0.022(0.026)$ \\
\hline & rs 864265 & $3.7 \pm 4.1$ & $6.1 \pm 16.9$ & 2.7 & $0.075(0.038)$ & $0.050(0.034)$ & $3.1 \pm 3.9$ & $2.4 \pm 1.8$ & $5.4 \pm 3.5$ & $-0.030(0.035)$ & $0.39(0.50)$ \\
\hline & rs 182052 & $3.5 \pm 3.7$ & $3.5 \pm 3.5$ & $5.3 \pm 12.5$ & $-0.047(0.018)$ & $0.011(0.0057)$ & $3.3 \pm 4.4$ & $3.2 \pm 3.9$ & $2.7 \pm 2.5$ & $0.029(0.017)$ & $0.092(0.083)$ \\
\hline & rs 17366568 & - & $4.4 \pm 7.1$ & $4.0 \pm 7.1$ & $0.019(0.061)$ & $0.76(0.92)$ & $9.1 \pm 0$ & $2.7 \pm 2.6$ & $3.1 \pm 3.8$ & $0.012(0.052)$ & $0.82(0.79)$ \\
\hline & rs6773957 & $3.7 \pm 3.9$ & $3.8 \pm 8.9$ & $4.8 \pm 6.9$ & $0.029(0.018)$ & $0.11(0.16)$ & $3.3 \pm 4.3$ & $2.9 \pm 3.6$ & $3.0 \pm 2.9$ & $-0.015(0.017)$ & $0.38(0.41)$ \\
\hline \multirow{7}{*}{$\begin{array}{l}\text { Adiponectin } \\
(\mu \mathrm{g} / \mathrm{mL})\end{array}$} & rs6810075 & $5.7 \pm 2.7$ & $6.1 \pm 3.1$ & $6.3 \pm 3.6$ & $-0.015(0.014)$ & $0.25(0.26)$ & $8.7 \pm 3.7$ & $10.0 \pm 5.2$ & $10.3 \pm 5.4$ & $-0.025(0.013)$ & $0.050(\mathbf{0 . 0 4 7})$ \\
\hline & rs 10937273 & $7.0 \pm 4.4$ & $6.2 \pm 3.2$ & $5.7 \pm 2.5$ & $-0.039(0.014)$ & $0.0058(0.0060)$ & $10.8 \pm 5.9$ & $10.3 \pm 5.1$ & $8.8 \pm 4.3$ & $-0.042(0.013)$ & $0.0015(0.0018)$ \\
\hline & rs1648707 & $6.6 \pm 4.0$ & $6.2 \pm 3.2$ & $5.6 \pm 2.5$ & $-0.032(0.014)$ & $0.018(0.019)$ & $10.6 \pm 5.8$ & $10.2 \pm 5$ & $8.7 \pm 4.3$ & $-0.035(0.013)$ & $0.0059(0.0064)$ \\
\hline & rs 864265 & $6.1 \pm 3.2$ & $5.8 \pm 2.8$ & - & $-0.025(0.028)$ & $0.36(0.34)$ & $9.8 \pm 5.0$ & $9.6 \pm 5.0$ & $10.9 \pm 5.8$ & $-0.013(0.025)$ & $0.61(0.65)$ \\
\hline & rs182052 & $5.6 \pm 2.5$ & $6.2 \pm 3.2$ & $6.3 \pm 3.6$ & $-0.021(0.013)$ & $0.12(0.13)$ & $8.7 \pm 3.7$ & $10 \pm 5.2$ & $10.3 \pm 5.4$ & $-0.026(0.013)$ & $0.042(0.036)$ \\
\hline & rs17366568 & - & $7.0 \pm 3.1$ & $6.0 \pm 3.1$ & $0.083(0.047)$ & $0.077(0.075)$ & - & $10.8 \pm 6.8$ & $9.7 \pm 4.9$ & $0.013(0.039)$ & $0.73(0.72)$ \\
\hline & rs6773957 & $5.8 \pm 2.4$ & $6.3 \pm 3.4$ & $6.2 \pm 3.7$ & $0.002(0.013)$ & $0.87(0.84)$ & $9.7 \pm 4.9$ & $9.7 \pm 4.6$ & $10.1 \pm 6.0$ & $0.004(0.012)$ & $0.77(0.68)$ \\
\hline
\end{tabular}

Data are expressed as means \pm SDs. Allele1, allele2, and the tested (adiponectin-decreasing) allele of each SNP are indicated in Table 2. The effect sizes and $P$-values are derived from linear regression analysis. Variables were adjusted for age and log-transformed BMI. Fasting plasma glucose, insulin, HOMA-IR, and adiponectin were log-transformed for the analysis. *In parentheses, $P$-values further adjusted for the treatment for type 2 diabetes. 11 , allele1/allele1; 12, allele1/allele2; 22, allele2/allele2. Numbers in bold indicate $P$-values less than 0.05 .

Because medications have an effect on adiposity, fasting plasma glucose, insulin, HOMA-IR, and adiponectin, we have analyzed these parameters adjusted for the treatment with diabetic medications (Tables 4 and 5). We found similar significant associations of the rs 10937273 and rs1648707 genotype with increased HOMA-IR and decreased adiponectin levels in women.

\section{Discussion}

Adiponectin is specifically secreted from adipose tissue, and its plasma levels are decreased during visceral fat accumulation $[2,4]$. Decreasing adiponectin levels are thought to be associated with the development of insulin resistance and type 2 diabetes $[4,5]$.
GWASs have shown that SNPs in $A D I P O Q$ have a high degree of association with adiponectin levels [6-13]; however, until now, the association between $A D I P O Q$ SNPs and insulin resistance in the context of visceral fat accumulation had not been investigated. In the current study, we addressed this question by analyzing parameters related to visceral fat levels; however, no SNPs were found to be associated with BMI, VFA, SFA, or the V/S ratio. Consistent with this, two previous studies reported that there were no significant associations between $A D I P O Q$ SNPs and VFA [22, 23]. Thus, taken together, previous data and our current analysis suggested that $A D I P O Q$ SNPs are not associated with VFA.

In this study, we replicated the association between 
Table 5 Replication analysis in the second set of participants

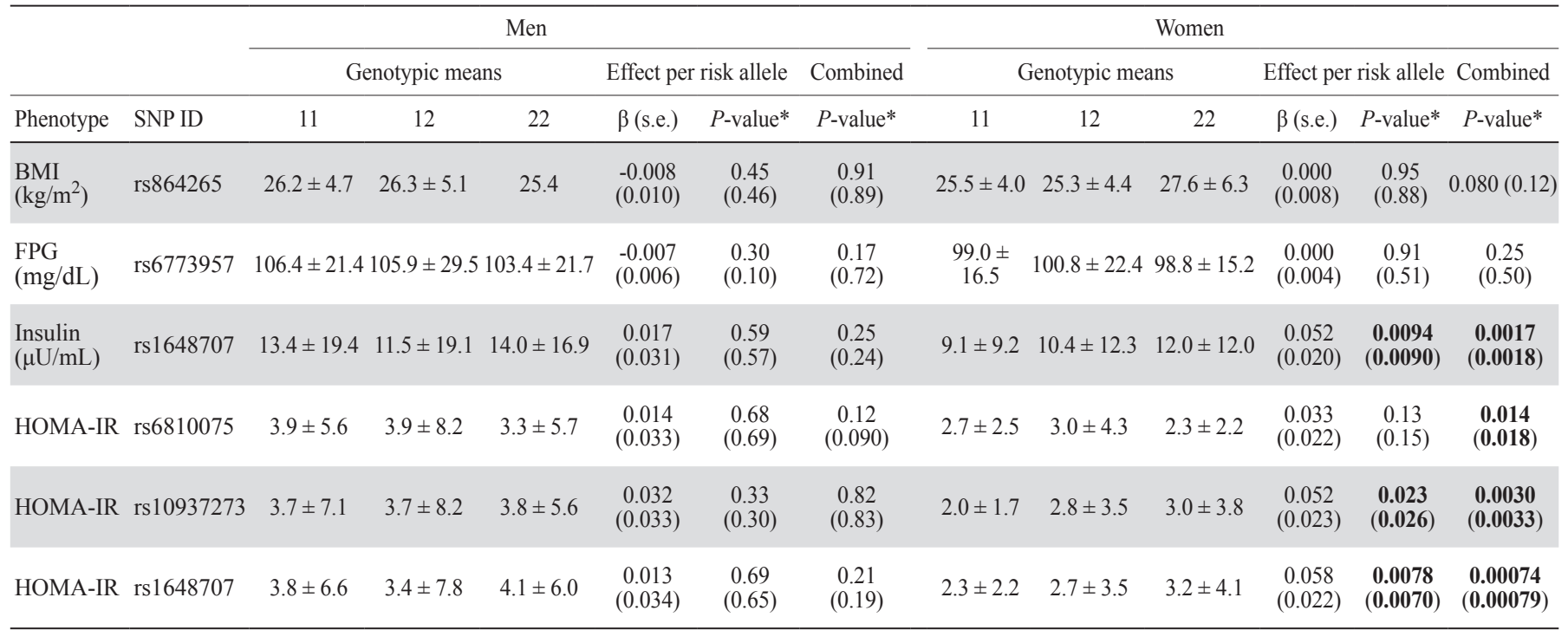

Data are expressed as means \pm SDs. Allele1, allele2, and the risk (adiponectin-decreasing) allele of each SNP are indicated in Table 2. The effect sizes and $P$-values are derived from linear regression analysis. Variables were adjusted for age and log-transformed BMI. FPG, insulin, and HOMA-IR were $\log$-transformed for the analysis. FPG, fasting plasma glucose; *In parentheses, $P$-values further adjusted for the treatment for type 2 diabetes. 11, allele1/allele1; 12, allele1/allele2; 22, allele2/allele2. Numbers in bold indicate $P$-values less than 0.05 .

rs10937273 and rs1648707, and adiponectin levels. We showed that rs10937273 and rs1648707 were significantly associated with HOMA-IR and that rs1648707 was significantly associated with fasting insulin in women, but not in men. Since these SNPs were not associated with BMI or VFA, the effects of these alleles on insulin levels and HOMA-IR would be independent of fat accumulation. The adiponectin-decreasing allele of $A D I P O Q$ (rs1648707) has been shown to be associated with increased insulin levels $(P<0.05)$ in Caucasians [8]. A recent meta-analysis of polymorphisms showed that G276T (rs1501299) is associated with insulin resistance only in Asians [29]. According to the HapMap database, G276T is in linkage disequilibrium (LD) with rs6773957 $\left(\mathrm{D}^{\prime}=1.000, \mathrm{r}^{2}=0.444\right)$. G276T is in weak LD with $\mathrm{rs} 10937273\left(\mathrm{D}^{\prime}=0.065\right.$, $\left.r^{2}=0.001\right)$ and rs1648707 $\left(D^{\prime}=0.039, r^{2}=0.000\right)$. In our study, the SNP rs6773957 (3'-untranslated region; 3'-UTR) was not associated with adiponectin, insulin levels, or HOMA-IR in men or women. The LD was different in the 5'-flanking region from the coding regions of the $A D I P O Q$ gene (Supplementary Fig. 1) [30]. Our results indicated that variations existing in the 5'-region of $A D I P O Q$ would be important for decreasing adiponectin levels and the development of insulin resistance in women. However, few reports have analyzed the association between $A D I P O Q$ SNPs and insulin resistance in men and women separately. Further studies in women are necessary to clarify the effects of $A D I P O Q$ SNPs on insulin resistance.

In summary, we found that two SNPs (rs10937273 and rs1648707) in the regulatory region of $A D I P O Q$ were associated with decreased adiponectin levels and insulin resistance independent of visceral fat accumulation. These SNPs could affect insulin sensitivity through the regulation of adiponectin production from adipose tissue in women.

\section{Acknowledgments}

This work was supported by a Grant-in-Aid from the Ministry of Education, Science, Sports, and Culture of Japan (25461343 to K.H., 26460999 to T.K., and 26870290 to A.K.). The authors express their appreciation to Drs. Ryoya Komatsu and Naoto Itoh for collection and data acquisition of samples.

\section{Disclosure Statement}

None of the authors has any potential conflicts of interest associated with this research. 
Supplementary Table 1 Association between $A D I P O Q$ haplotypes and HOMA-IR in women

\begin{tabular}{lcccc}
\hline SNPs & Haplotype & frequency & $P$-value & $\begin{array}{c}P \text {-value } \\
\text { adjusted for treatment }\end{array}$ \\
\hline rs10937273 & GC & 0.575 & 0.0011 & 0.0012 \\
rs1648707 & AA & 0.352 & Reference & Reference \\
& GA & 0.070 & 0.93 & 0.94 \\
\hline rs6810075 & CGC & 0.481 & 0.0039 & 0.0045 \\
rs10937273 & TAA & 0.352 & Reference & Reference \\
rs1648707 & TGC & 0.094 & 0.013 & 0.011 \\
& TGA & 0.068 & 0.91 & 0.92 \\
\hline rs6810075 & CGCGA & 0.481 & 0.0059 & 0.0068 \\
rs10937273 & TAAGG & 0.351 & Reference & Reference \\
rs1648707 & TGCGG & 0.093 & 0.0088 & 0.0078 \\
rs864265 & TGATG & 0.068 & 0.89 & 0.90 \\
\hline
\end{tabular}

The program Haplo.Stats was used to explore associations of $A D I P O Q$ haplotypes and HOMA-IR.

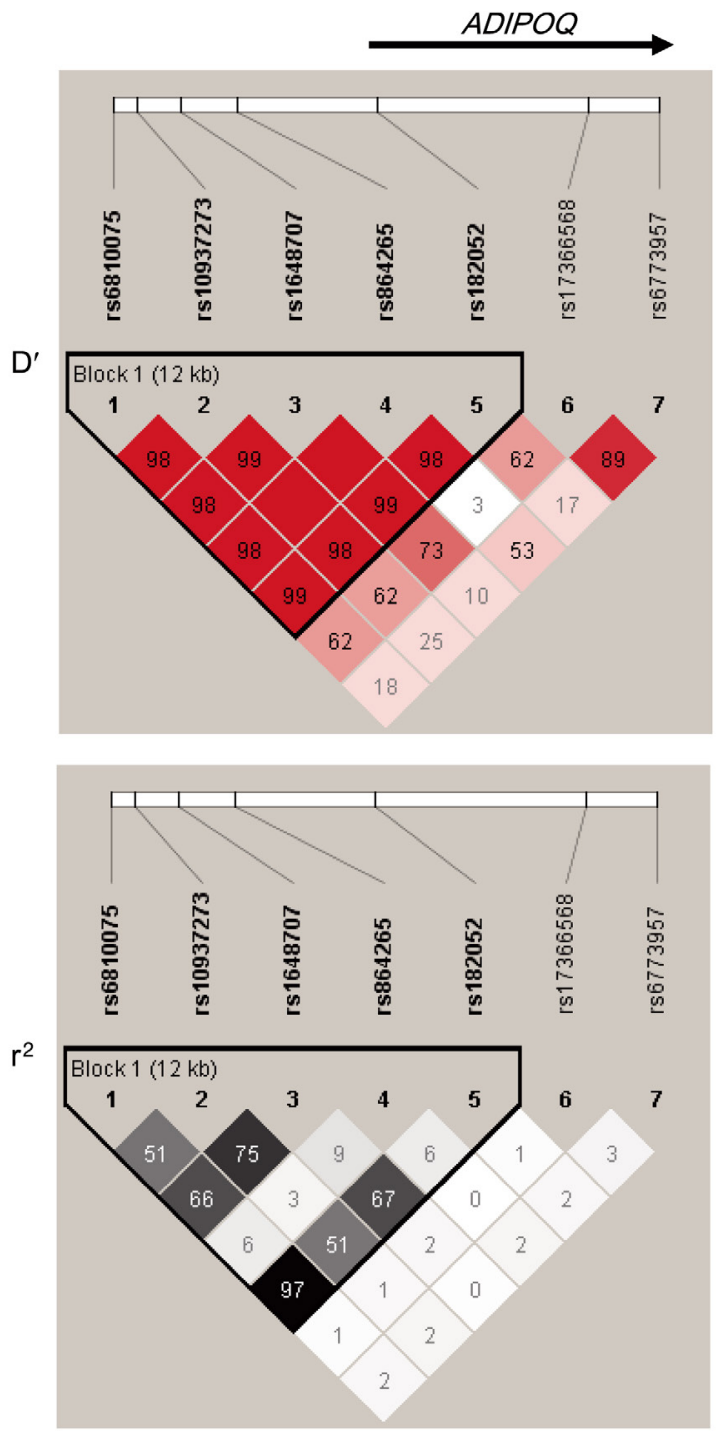

Supplementary Fig. 1 LD map of seven SNPs in ADIPOQ 


\section{References}

1. Carr DB, Utzschneider KM, Hull RL, Kodama K, Retzlaff BM, et al. (2004) Intra-abdominal fat is a major determinant of the National Cholesterol Education Program Adult Treatment Panel III criteria for the metabolic syndrome. Diabetes 53: 2087-2094.

2. Matsuzawa Y (2006) Therapy insight: adipocytokines in metabolic syndrome and related cardiovascular disease. Nat Clin Pract Cardiovasc Med 3: 35-42.

3. Maeda K, Okubo K, Shimomura I, Funahashi T, Matsuzawa Y, et al. (1996) cDNA cloning and expression of a novel adipose specific collagen-like factor, apM1 (AdiPose Most abundant Gene transcript 1). Biochem Biophys Res Commun 221: 286-289.

4. Hotta K, Funahashi T, Bodkin NL, Ortmeyer HK, Arita Y, et al. (2001) Circulating concentrations of the adipocyte protein adiponectin are decreased in parallel with reduced insulin sensitivity during the progression to type 2 diabetes in rhesus monkeys. Diabetes 50: 11261133.

5. Hotta K, Funahashi T, Arita Y, Takahashi M, Matsuda $\mathrm{M}$, et al. (2000) Plasma concentrations of a novel, adipose-specific protein, adiponectin, in type 2 diabetic patients. Arterioscler Thromb Vasc Biol 20: 1595-1599.

6. Ling H, Waterworth DM, Stirnadel HA, Pollin TI, Barter PJ, et al. (2009) Genome-wide linkage and association analyses to identify genes influencing adiponectin levels: the GEMS Study. Obesity (Silver Spring) 17: 737-744.

7. Heid IM, Henneman P, Hicks A, Coassin S, Winkler T, et al. (2010) Clear detection of ADIPOQ locus as the major gene for plasma adiponectin: results of genomewide association analyses including 4659 European individuals. Atherosclerosis 208: 412-420.

8. Richards JB, Waterworth D, O'Rahilly S, Hivert MF, Loos RJ, et al. (2009) A genome-wide association study reveals variants in ARL15 that influence adiponectin levels. PLoS Genet 5: e1000768.

9. Wu Y, Li Y, Lange EM, Croteau-Chonka DC, Kuzawa CW, et al. (2010) Genome-wide association study for adiponectin levels in Filipino women identifies CDH13 and a novel uncommon haplotype at KNG1-ADIPOQ. Hum Mol Genet 19: 4955-4964.

10. Jee SH, Sull JW, Lee JE, Shin C, Park J, et al. (2010) Adiponectin concentrations: a genome-wide association study. Am J Hum Genet 87: 545-552.

11. Dastani Z, Hivert MF, Timpson N, Perry JR, Yuan X, et al. (2012) Novel loci for adiponectin levels and their influence on type 2 diabetes and metabolic traits: a multi-ethnic meta-analysis of 45,891 individuals. PLoS Genet 8: e1002607.

12. Wu Y, Gao H, Li H, Tabara Y, Nakatochi M, et al. (2014) A meta-analysis of genome-wide association studies for adiponectin levels in East Asians identifies a novel locus near WDR11-FGFR2. Hum Mol Genet 23: 1108-1119.

13. Peters KE, Beilby J, Cadby G, Warrington NM, Bruce DG, et al. (2013) A comprehensive investigation of variants in genes encoding adiponectin (ADIPOQ) and its receptors (ADIPOR1/R2), and their association with serum adiponectin, type 2 diabetes, insulin resistance and the metabolic syndrome. BMC Med Genet 14: 15.

14. Mather KJ, Christophi CA, Jablonski KA, Knowler WC, Goldberg RB, et al. (2012) Common variants in genes encoding adiponectin (ADIPOQ) and its receptors (ADIPOR1/2), adiponectin concentrations, and diabetes incidence in the Diabetes Prevention Program. Diabet Med 29: 1579-1588.

15. Mastuzawa Y (2005) Metabolic syndrome - definition and diagnostic criteria in Japan. J Atheroscler Thromb 12: 301 .

16. Fox CS, Liu Y, White CC, Feitosa M, Smith AV, et al. (2012) Genome-wide association for abdominal subcutaneous and visceral adipose reveals a novel locus for visceral fat in women. PLoS Genet 8: e1002695.

17. Hotta K, Nakamura M, Nakamura T, Matsuo T, Nakata Y, et al. (2010) Polymorphisms in NRXN3, TFAP2B, MSRA, LYPLAL1, FTO and MC4R and their effect on visceral fat area in the Japanese population. J Hum Genet 55: 738-742.

18. Hotta K, Kitamoto T, Kitamoto A, Mizusawa S, Matsuo T, et al. (2011) Computed tomography analysis of the association between SH2B1 rs7498665 single-nucleotide polymorphism and visceral fat area. J Hum Genet 56: 716-719.

19. Hotta K, Kitamoto A, Kitamoto T, Mizusawa S, Teranishi H, et al. (2012) Association between type 2 diabetes genetic susceptibility loci and visceral and subcutaneous fat area as determined by computed tomography. J Hum Genet 57: 305-310.

20. Hotta K, Kitamoto A, Kitamoto T, Mizusawa S, Teranishi $\mathrm{H}$, et al. (2012) Genetic variations in the CYP17A1 and NT5C2 genes are associated with a reduction in visceral and subcutaneous fat areas in Japanese women. J Hum Genet 57: 46-51.

21. Hotta K, Kitamoto A, Kitamoto T, Mizusawa S, Teranishi $\mathrm{H}$, et al. (2013) Replication study of 15 recently published loci for body fat distribution in the Japanese population. J Atheroscler Thromb 20: 336-350.

22. An SS, Hanley AJ, Ziegler JT, Brown WM, Haffner SM, et al. (2012) Association between ADIPOQ SNPs with plasma adiponectin and glucose homeostasis and adiposity phenotypes in the IRAS Family Study. Mol Genet Metab 107: 721-728.

23. Loos RJ, Ruchat S, Rankinen T, Tremblay A, Pérusse L, et al. (2007) Adiponectin and adiponectin receptor gene variants in relation to resting metabolic rate, respi- 
ratory quotient, and adiposity-related phenotypes in the Québec Family Study. Am J Clin Nutr 85(1): 26-34.

24. Yoshizumi T, Nakamura T, Yamane M, Islam AH, Menju M, et al. (1999) Abdominal fat: standardized technique for measurement at CT. Radiology 211: 283286.

25. Ohnishi Y, Tanaka T, Ozaki K, Yamada R, Suzuki H, et al. (2001) A high-throughput SNP typing system for genome-wide association studies. J Hum Genet 46: 471-477.

26. Nielsen DM, Ehm MG, Weir BS (1998) Detecting marker-disease association by testing for Hardy-Weinberg disequilibrium at a marker locus. Am J Hum Genet 63: 1531-1540.

27. Kotani K, Tokunaga K, Fujioka S, Kobatake T, Keno Y, et al. (1994) Sexual dimorphism of age-related changes in whole-body fat distribution in the obese. Int $J$ Obes Relat Metab Disord 18: 207-202.

28. Nagaretani H, Nakamura T, Funahashi T, Kotani K, Miyanaga M, et al. (2001) Visceral fat is a major contributor for multiple risk factor clustering in Japanese men with impaired glucose tolerance. Diabetes Care 24: 2127-2133.

29. Ouyang S, Cao D, Liu Z, Ma F, Wu J (2014) Metaanalysis of the association of ADIPOQ G276T polymorphism with insulin resistance and blood glucose. Endocrine 47: 749-757.

30. Tanimura D, Shibata R, Izawa H, Hirashiki A, Asano H, et al. (2011) Relation of a common variant of the adiponectin gene to serum adiponectin concentration and metabolic traits in an aged Japanese population. Eur $J$ Hum Genet 19: 262-269. 